\title{
p53 Expression in Surface Epithelial Ovarian Tumors and its Correlation with Histological Type and Grade of the Tumor
}

\author{
Authors \\ Dr Abhilash Singh ${ }^{1}$, Dr Neelam Sharma ${ }^{2}$, Dr Lalita Negi ${ }^{3}$, Dr Rajeev Sood \\ ${ }^{1}$ Junior Resident, Department of Pathology, IGMC, Shimla \\ ${ }^{2}$ Professor, Department of Pathology, IGMC, Shimla \\ ${ }^{3}$ Associate Professor, Department of Pathology, IGMC, Shimla \\ ${ }^{4}$ Professor, Department of Obstetrics and Gynaecology, IGMC Shimla \\ *Corresponding author \\ Dr Neelam Sharma
}

\section{Introduction}

Ovarian cancer is the sixth most common female cancer worldwide ${ }^{1}$ and accounts for a significant number of deaths from malignancies of the female genital tract ${ }^{2}$. It is considered to be the most lethal gynaecological malignancy ${ }^{10}$. Its incidence rates are higher in developed than developing countries. The age standardized incidence rates of ovarian cancer for 2020 in world and India is 6.6 and 6.7 respectively. In most of the population-based cancer registries in India, ovarian cancer is the third leading site of cancer among women, trailing behind cervix and breast cancer. Indian Cancer Registry data project ovary as an important site of cancer in women, comprising up to $8.7 \%$ of cancers in different parts of the country. ${ }^{9}$

About $80 \%$ ovarian tumours are benign and occur in younger age group comprising of 20-45 years however, malignant tumours are more common in 40-65years. Important etiological risk factors are increasing age, positive family history, increase age of reproduction, high socio-economic classes, and nulliparity ${ }^{11}$.
World Health Organization (WHO) categorizes primary ovarian neoplasms according to histogenetic principles, mainly with regards to their derivation i.e. from coelomic surface epithelial cells, germ cells and mesenchyme (sex cord and stroma). In addition to primary benign and malignant neoplasms, there are also borderline tumours which represent non-invasive tumours of uncertain malignant potential. $^{10}$ Surface epithelial tumors of the ovary account for approximately two-third of all ovarian tumors and their malignant forms represent about $90 \%$ of ovarian cancers. ${ }^{7}$

Based on their morphological features surface epithelial tumors of the ovary are categorized as serous, mucinous, endometrioid, clear cell, transitional cell, mixed epithelial cells and undifferentiated carcinomas. These subtypes differ significantly in their potential sites of origin, molecular signature, prognosis, and response to treatment. $^{3}$

Identification of new biological prognostic markers would be of great importance to select patients with a possibly favourable or poor clinical 
outcome and might help to improve treatment planning. ${ }^{1}$ Steroid hormones such as estrogen and progesterone are thought to play an important role in the process of carcinogenesis of ovarian tumors. Similarly Ki-67 is a proliferation marker helpful in predicting disease outcome in many types of malignancies including ovarian neoplasms. ${ }^{4}$ Regulators of apoptosis, especially p53 and Bcl-2 have also been studied as potential prognostic factors of epithelial ovarian cancer. ${ }^{1}$

One of the most studied prognostic markers in ovarian cancer so far is over expression of p53. Intranuclear accumulation of p53 has been detected in as many as $69 \%$ ovarian carcinomas by immunohistochemical studies. ${ }^{7}$ Although the nucleotide sequencing is the most reliable technique to detect gene mutation, it is labor intensive, time consuming, and therefore, currently has limited application in clinical pathology practice. Immunohistochemical analysis of p53 expression is therefore commonly used as a surrogate for mutational analysis. ${ }^{13}$

P53 gene located on the short arm of chromosome seventeen. ${ }^{8}$ It is a tumor suppressor gene which links cell damage with DNA repair, cell cycle arrest, and apoptosis. In view of these activities p53 has been rightfully called as "guardian of the genome."7 A cell with a damaged DNA that cannot be repaired is directed by P53 to undergo apoptosis. ${ }^{8}$ The ability of p53 to control apoptosis in response to DNA damage has important practical therapeutic implications. ${ }^{7}$ Irradiation and chemotherapy, the two common modalities of cancer treatment, mediate their effects by inducing DNA damage and subsequent apoptosis. ${ }^{8}$ Tumors that retain normal p53 are more likely to respond to irradiation and chemotherapy therapy than tumors that carry mutated alleles of the gene. Mutant p53 proteins have a prolonged half-life, accumulate in the nucleus, and can be detected by immunohistochemistry. ${ }^{7}$

However the clinical significance of p53 overexpression in patients with ovarian carcinoma is uncertain. Previous studies have yielded conflicting results. The biggest diagnostic dilemma in ovarian surface epithelial tumors is differentiating between borderline and invasive carcinomas. ${ }^{8}$

The aim of the present study is to evaluate p53 expression in surface epithelial ovarian tumours and its association with histomorphologic type and grade of tumours.

\section{Material and Methods}

This was a hospital based cross sectional observational study conducted for one year from $1^{\text {st }}$ June 2019 to $31^{\text {st }}$ May 2020 in the Department of Pathology, Indira Gandhi Medical College, Shimla. All the surface epithelial ovarian tumour specimens with definite histopathological diagnosis, irrespective of age were considered for study. Ovarian tumours other than surface epithelial ovarian tumours, metastatic tumours from non ovarian primary, patients with SEOTs on/prior radiation or chemotherapy and patients with recurrence of SEOTs were excluded from the study.

Study specimens were further evaluated with immunohistochemistry for the presence of p53 and its score. Positive and negative controls were run simultaneously with all patients' specimens. High grade serous carcinoma of ovary with known positivity was used as a positive control. Phosphate buffer solution instead of primary antibody was used as negative control. Positive expression on the immunostained slides was interpreted as the percentage of the positive tumour nuclei. Positivity was defined as brown reaction product in the nucleus. At least 10 random high power fields were counted with a minimum of 1000 cells. Sections were considered positive when at least $\geq 5 \%$ percentages of cells are positive for $\mathrm{p} 53$ receptor. Immunoreactivity for p53 was evaluated semi quantitatively according to the percentage of positive tumour nuclei, scored as follows:

\begin{tabular}{|c|c|c|}
\hline Grade & $\begin{array}{c}\text { Proportion/ } \\
\text { Positivity Score (PS) }\end{array}$ & $\begin{array}{c}\text { Percentage of } \\
\text { Stained Cells }\end{array}$ \\
\hline 0 & 0 & $<5 \%$ \\
\hline 1 & +1 & $5 \%-25 \%$ \\
\hline 2 & +2 & $25-75 \%$ \\
\hline 3 & +3 & $>75 \%$ \\
\hline
\end{tabular}


Statistical significance of correlation between positive p53 staining and tumor type and tumor grade was calculated by using EpiSoft software version 7 and chi square analysis. Significance was assumed at a $\mathrm{P}$ value less than 0.05 .

\section{Results}

Seventy six specimens of surface epithelial ovarian tumors were received in the

Department of Pathology. They were subjected to histopathological examination and immunohistochemistry for $\mathrm{p} 53$ protein. The age of the patients ranged from 21 to 90 years with mean age of 47 years. Youngest 3 patients were 21 years of age. Two of them were diagnosed as serous cystadenoma and one as mucinous cystadenoma. Oldest patient was 90 years old diagnosed with endometroid carcinoma. Most of patients were premenopausal $(55.3 \%)$ compared to postmenopausal patients $(44.7 \%)$. Majority of tumors were unilateral $(77.6 \%)$. Bilateral tumors were seen in only $22.4 \%$ percent. On gross appearance, most of the tumors $(55.3 \%)$ were cystic, $7.9 \%$ were solid and $36.8 \%$ tumors revealed both solid and cystic areas. Fifty four percent cystic tumors were uniloculated and $46 \%$ were multiloculated.

Histological classification was done in accordance with the WHO classification of ovarian tumors (2014). Serous tumors $42(55.3 \%)$ were the most frequent tumors encountered in the study followed by mucinous tumors $24(31.6 \%)$. Transitional cell tumors and endometroid tumors accounted for $4(5.3 \%)$ cases in each group.

Tumors were further categorized as benign, borderline and malignant based upon their biological behavior. Majority of tumors were benign (50\%) followed by malignant $(42.1 \%)$ and borderline tumors $(7.9 \%)$. Serous cyst adenomas $(47.4 \%)$ constituted the predominant group among the benign tumors followed by mucinous cyst adenomas (44.7\%) and Brenner tumors (7.9\%).Mean age of presentation in benign tumors was 46 years.

Borderline tumors accounted for 6 cases (7.9\%) and comprised of 4 atypical proliferative mucinous tumors (APMT) and 2 atypical proliferative serous tumors (APST). Mean age of presentation in borderline tumors was 46.1 years.

Amongst malignant tumors, serous carcinoma constituted $71.9 \%$ of total cases followed by endometroid carcinomas (12.5\%), mucinous carcinomas $(9.4 \%)$ and 1 case each of malignant Brenner tumor and clear cell carcinoma. Mean age for presentation in malignant tumors was 47.1years.

Twenty eight malignant tumors were graded according to "two tier grading system" as low and high grade tumors. Among them 23(82.1\%) were high grade while $5(17.9 \%)$ were low grade tumor. p53 immunostaining was perfomed on all 76 cases, carried out on Biogenex Xmatrix Fully Automated Front-end Processing System as per the standard protocol. Thirty four $(44.7 \%)$ out of total 76 SEOTs showed positivity for p53 immunostaining.

Positive expression was interpreted as the percentage of the positive tumour nuclei, when at least $\geq 5 \%$ nuclei showed p53 immunostaining. Amongst 34 cases with positive p53 expression $21.1 \%$ showed +3 IHC score $(>75 \%)$ followed by $18.42 \%$ cases with +2 IHC score $(25-75 \%)$. Only $5.3 \%$ cases revealed +1 IHC score $(5-25 \%)+3$ IHC score was observed mainly in malignant tumors. Majority of which were high grade serous carcinomas 10/16 (62.5\%). +2 IHC score was observed in 8 malignant tumors (3 cases of mucinous carcinoma, 2 cases each of serous and endometroid carcinoma and one case malignant brenner tumor), one case of APST and 5 benign tumors. Whereas +1 IHC score was observed in one case of low grade serous carcinoma and 3 benign tumors including two mucinous cyst adenomas and one benign Brenner tumor.

p53 positivity was reported to be higher $(53.9 \%)$ in $5^{\text {th }}$ decade or later compared to only $25 \%$ in 21 39 years age group. Age group showed statistical significant correlation with p53 positivity ( $\mathbf{P}=\mathbf{0 . 0 1 8})$.

Expression was seen more in postmenopausal 


\section{JMSCR Vol||09||Issue||04||Page 36-46||April}

women $(61.8 \%)$ than in premenopausal women $(30.95 \%)$ and showed statistical significant correlation with p53 positivity ( $\mathbf{p = 0 . 0 0 7 2}$ ).

In our study p53 protein expression was found to be more in malignant tumors $(71.9 \%)$ as compared to borderline (50\%) and benign tumors (21.1\%). Tumor type showed statistical significant correlation with $\mathrm{p} 53$ positivity $(\mathbf{p}=\mathbf{0 . 0 0 0 1})$.

Immunoreactivity was higher in serous tumors (45.2\%) compared to mucinous tumors with positivity in only $29.2 \%$ cases. Three out of four $(75 \%)$ transitional cell tumors revealed immunostainingfor p53 expression. All four cases of endometroid tumors were p53 positive. One case of clear cell tumor encountered in the study also expressed p53 protein.

Eightout of total 38 benign tumors positive for p53 expression included 3 cases each of serous and mucinous cystadenoma and 2 cases of benign Brenner tumor. One case of seromucinous cystadenoma was negative for p53 expression.

Amongst borderline tumors, both atypical proliferative serous tumors (APSTs) and only one out three atypical proliferative mucinous tumors (APMTs) showed $\mathrm{p} 53$ positivity.

Among malignant tumors, 14/23 (60.9\%) cases of serous carcinomas, all the cases of mucinous carcinoma, malignant Brenner tumor, endometroid carcinoma and clear cell carcinoma expressed p53 protein. Protein expression was reported in all low grade tumors $(100 \%)$ and in $60.9 \%$ of high grade tumors $(\mathbf{P}=\mathbf{0 . 0 8 9})$.

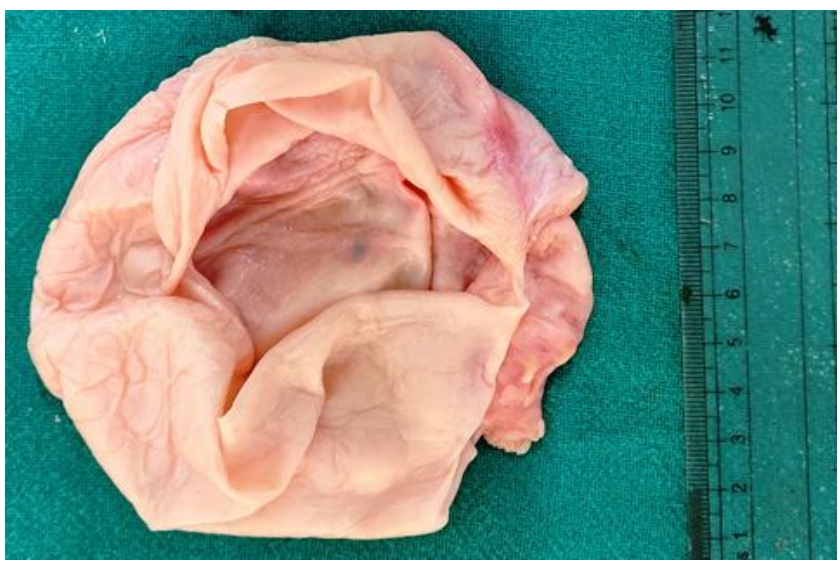

Serous Cystadenoma

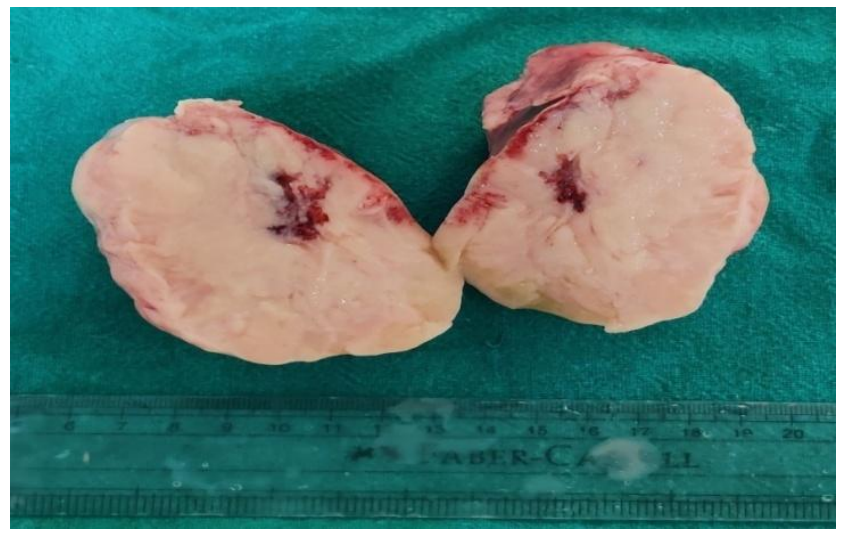

Brenner Tumor

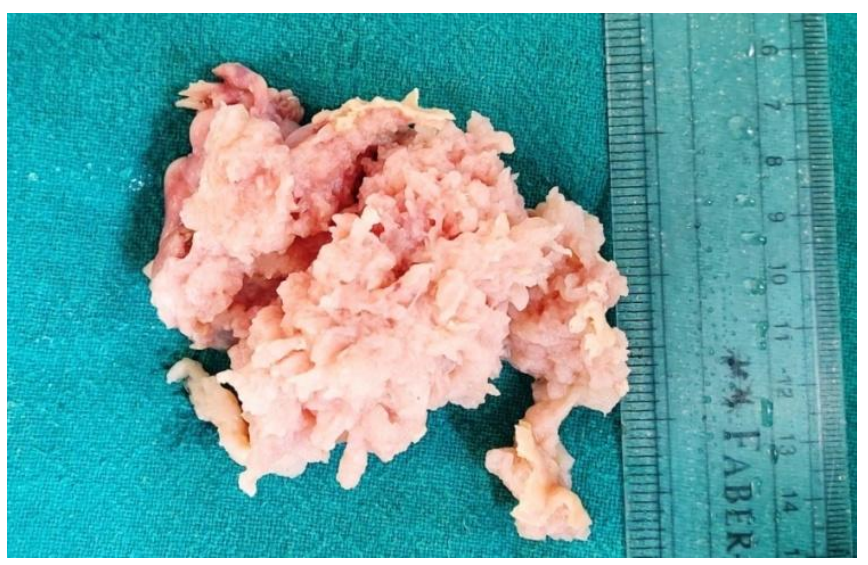

Atypical Proliferative Serous Tumor

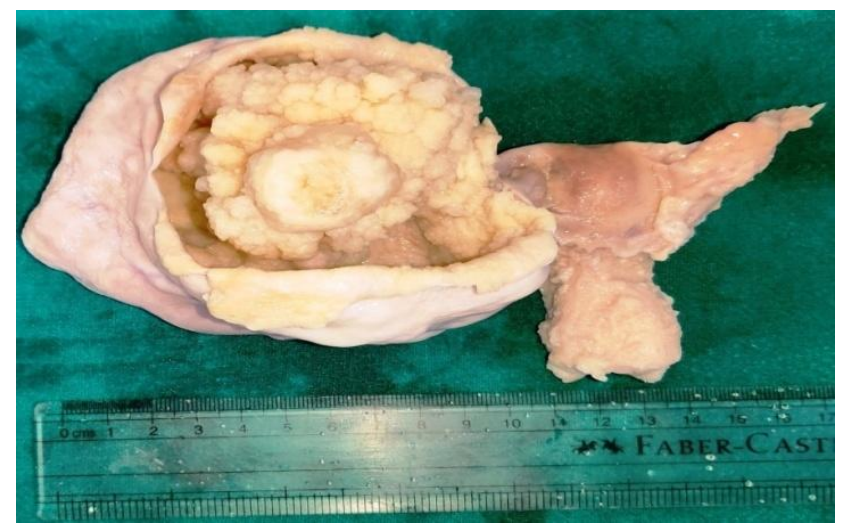

Serous Carcinoma

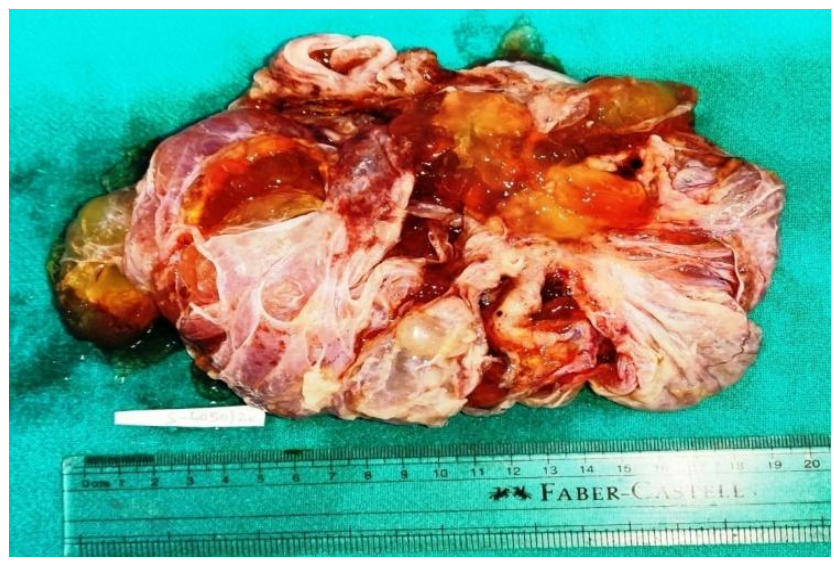

Mucinous Carcinoma 


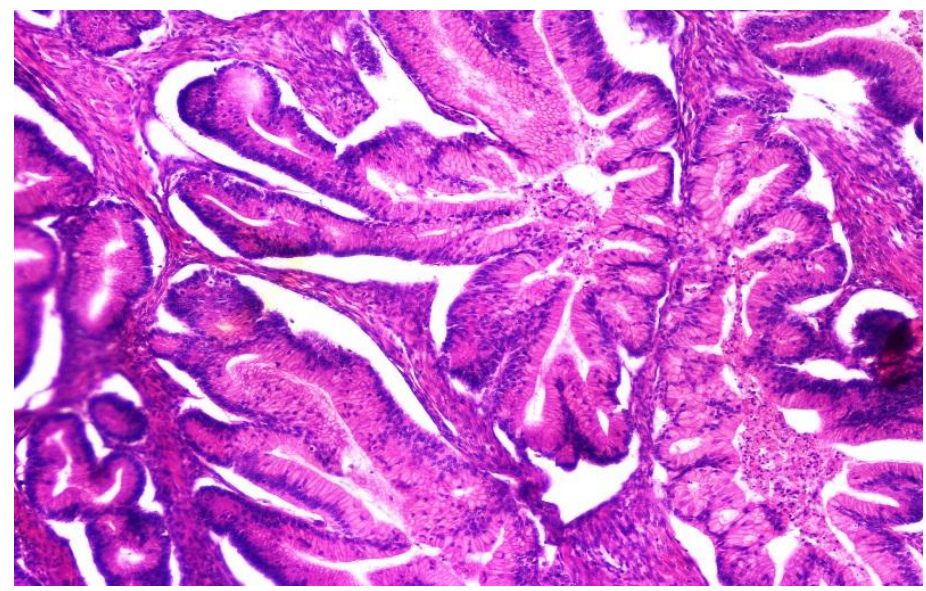

Atypical Proliferative Mucinous Tumor X 40 (H\&E)

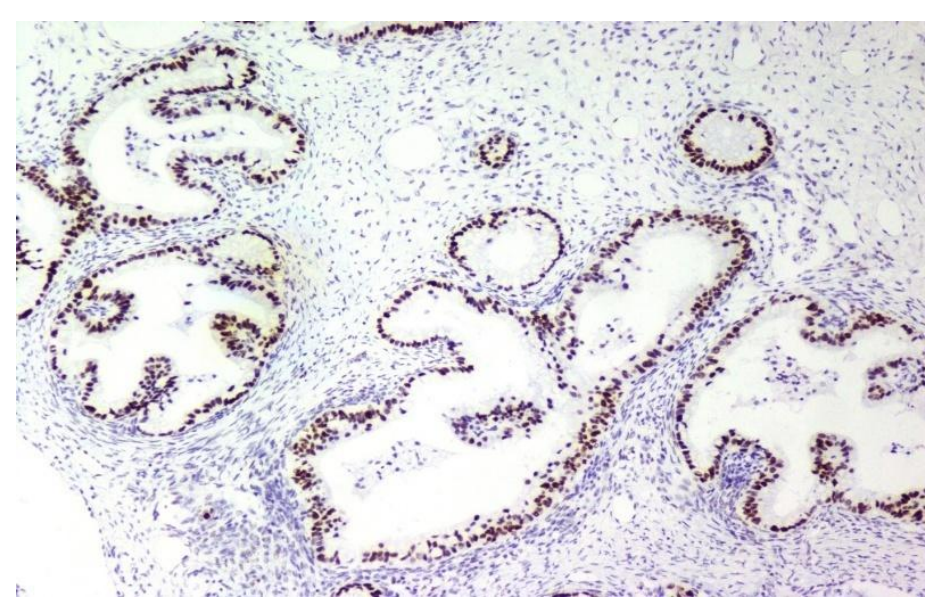

p53 Positivity in Atypical Proliferative Mucinous Tumor (X 40)

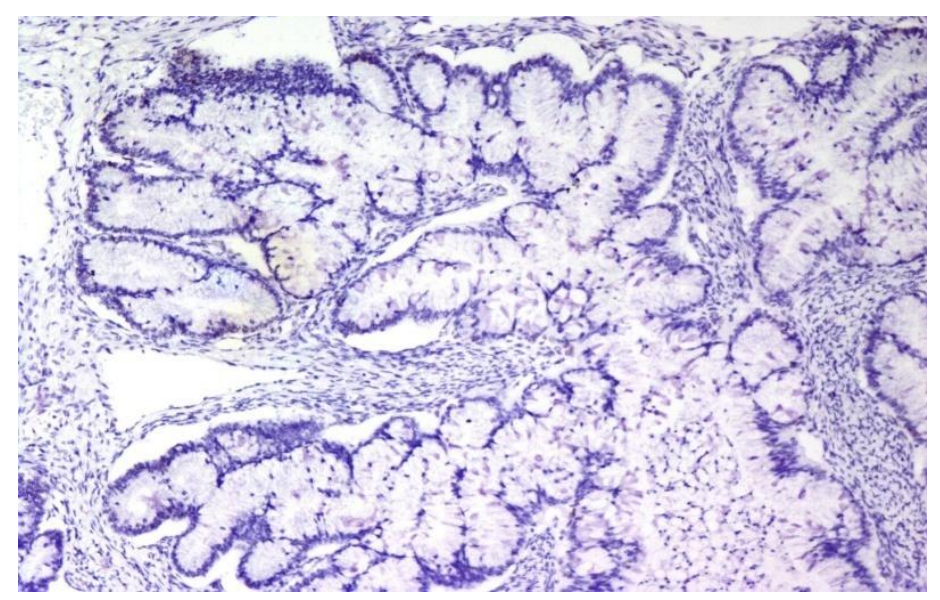

p53 Negativity in Atypical Proliferative Mucinous Tumor (X 100)

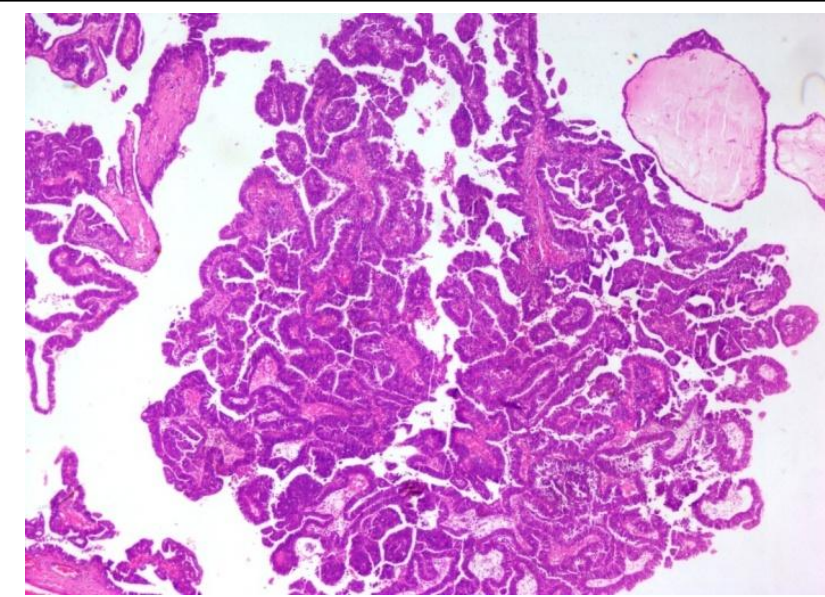

Atypical Proliferative Serous Tumor X 40 (H\&E)

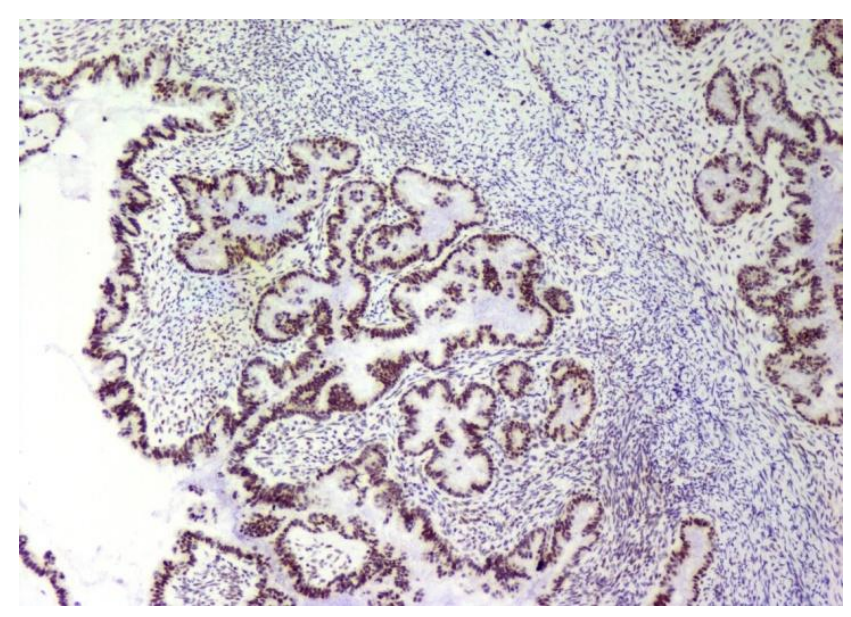

p53 Positivity in Atypical Proliferative Serous Tumor (X 100)
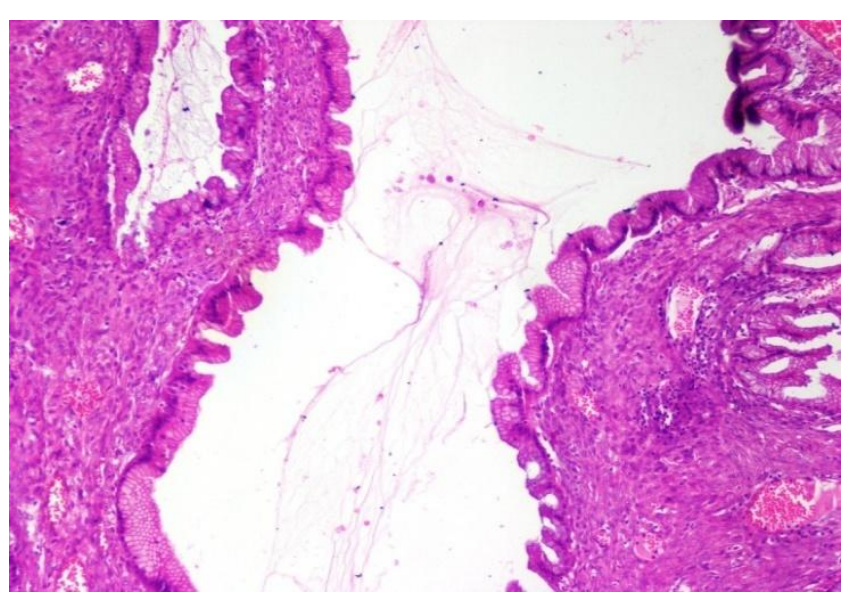

Benign Mucinous Cystadenoma X 100 (H\&E) 


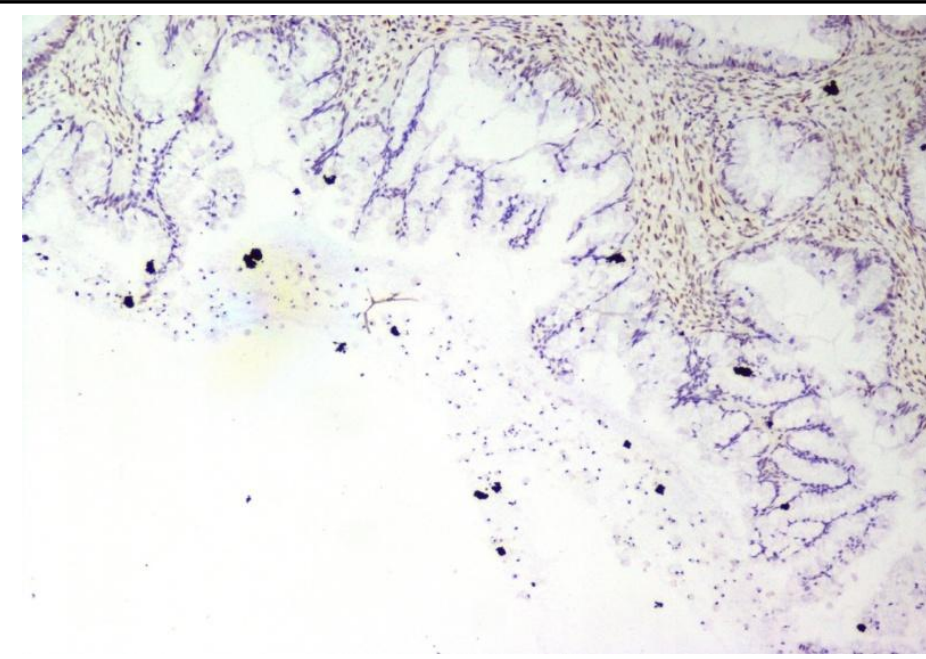

p53 Negativity in Mucinous Cystadenoma ( $X$ 100)

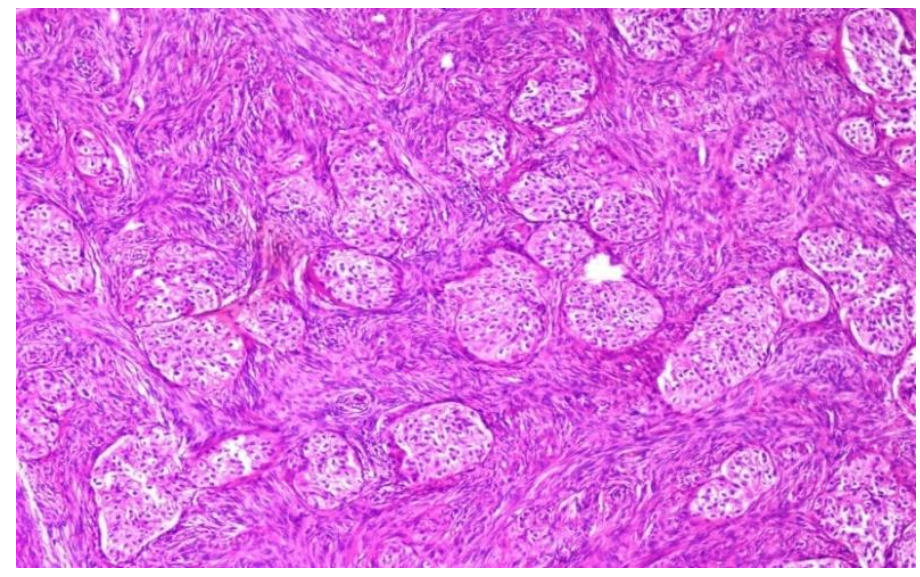

Benign Brenner Tumor: Round to ovoid nests of transitional cells surrounded by fibrotic stroma X 100 (H\&E)

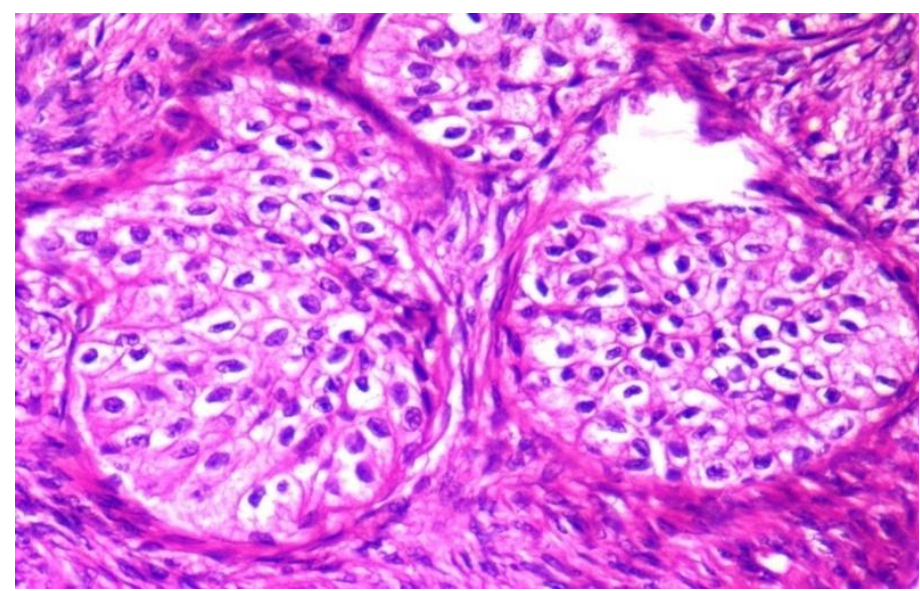

Benign Brenner Tumor X 400 (H\&E)

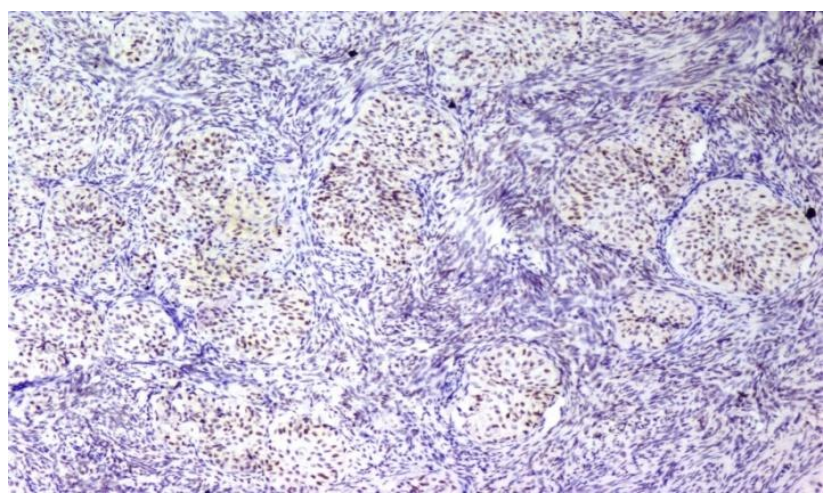

p53 Positivity in Benign Brenner Tumor (X 100)

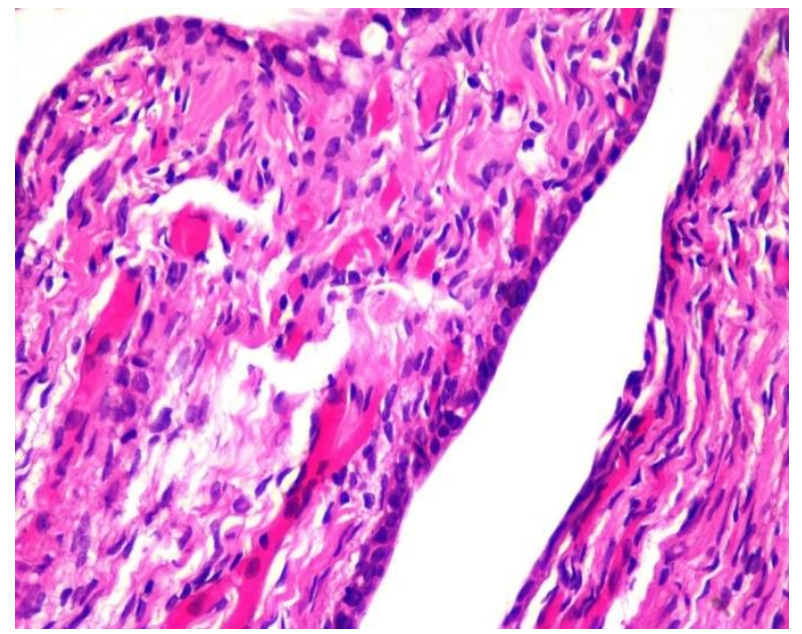

Serous Cystadenoma: Lined by single layered cuboidal serous epithelial cells. X 400 (H\&E)

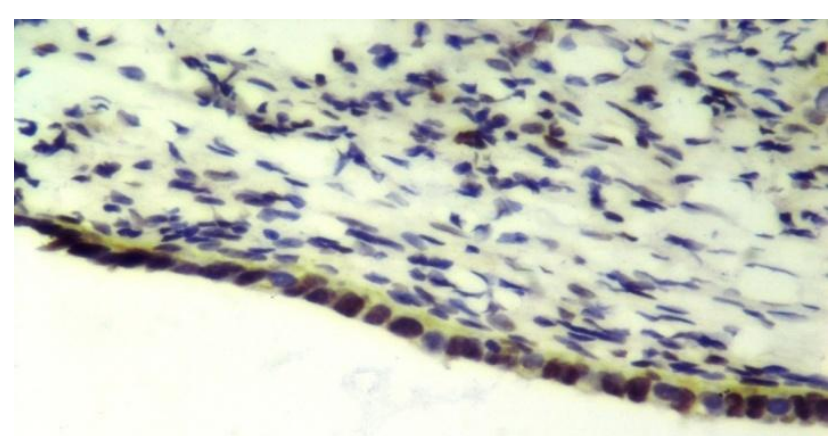

p53 Positivity in Serous Cystadenoma (X 400) 


\section{JMSCR Vol||09||Issue||04||Page 36-46||April}

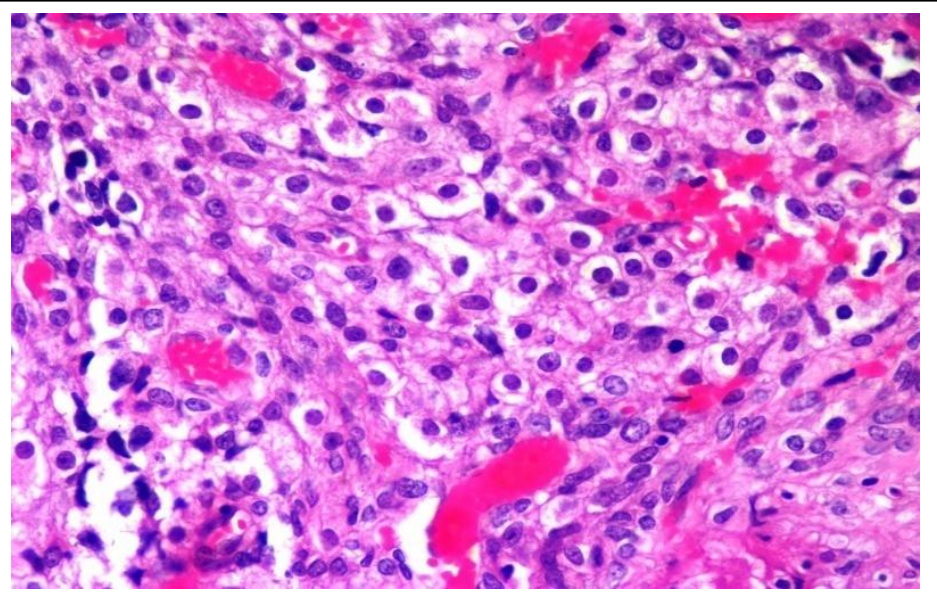

CLEAR CELL CARCINOMA: Solid pattern composed of cells with clear to finely granular cytoplasm X 400 (H\&E)

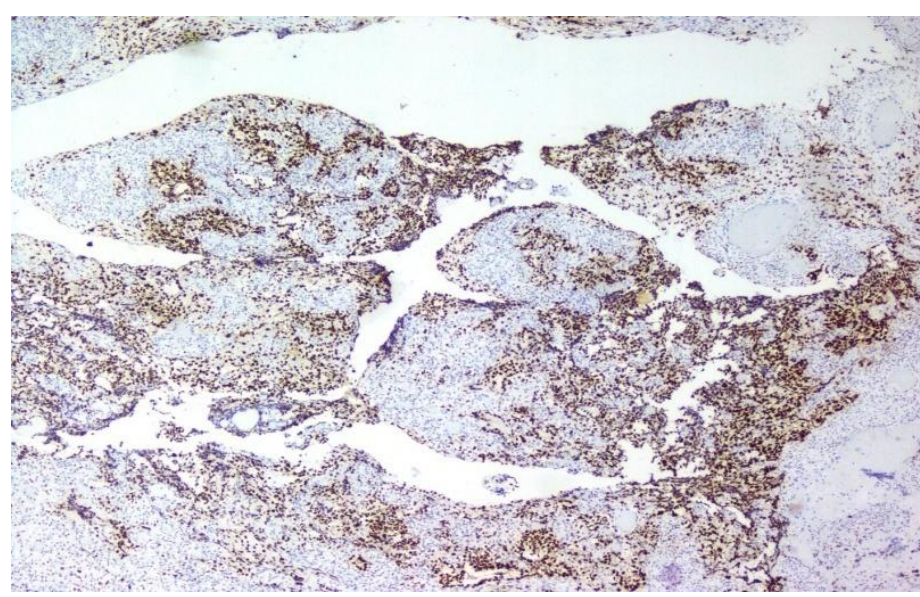

p53 Positivity In Clear Cell Carcinoma (X 40)

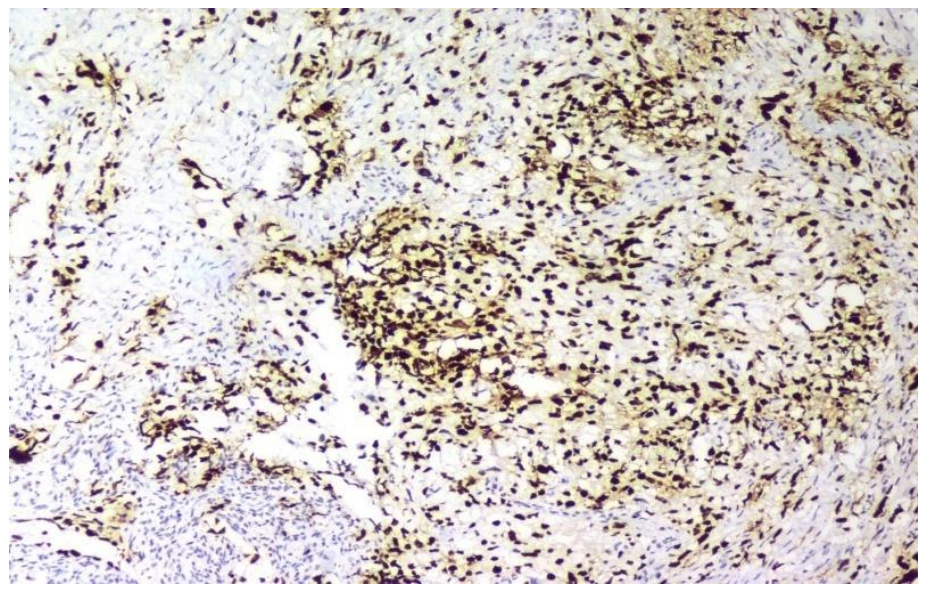

p53 Positivity in Clear Cell Carcinoma (X 100)

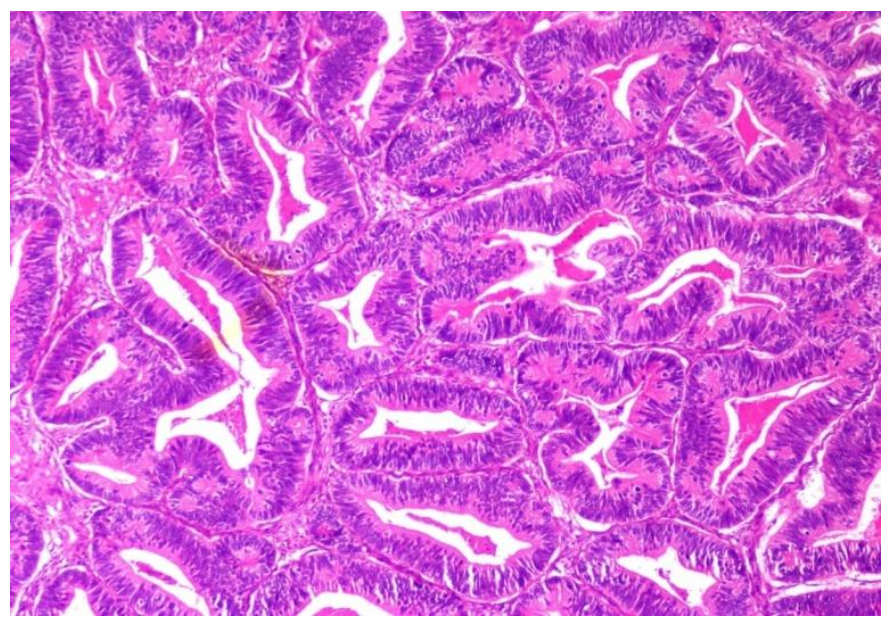

Endometroid Carcinoma: Back to back malignant glands X 100 (H\&E)

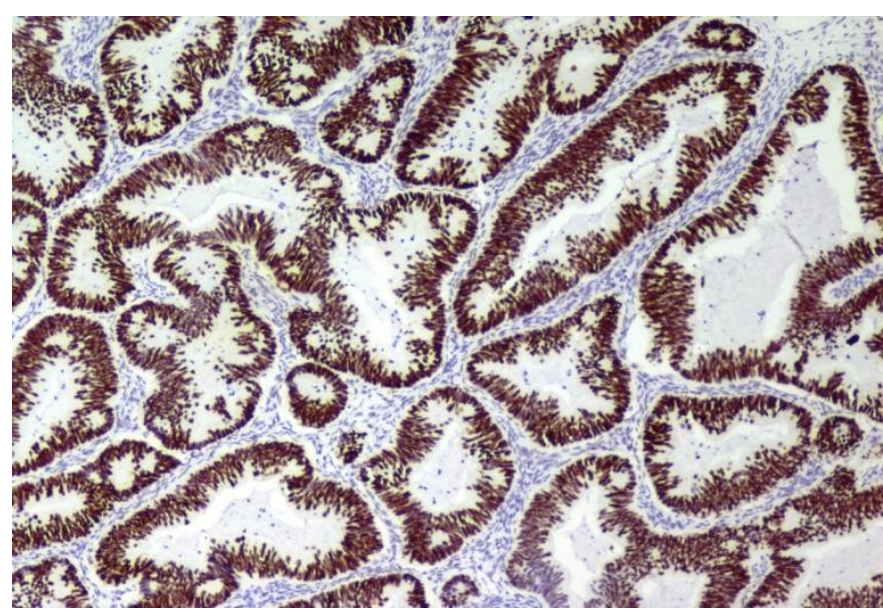

p53 Positivity in Endometroid Carcinoma (X 400)

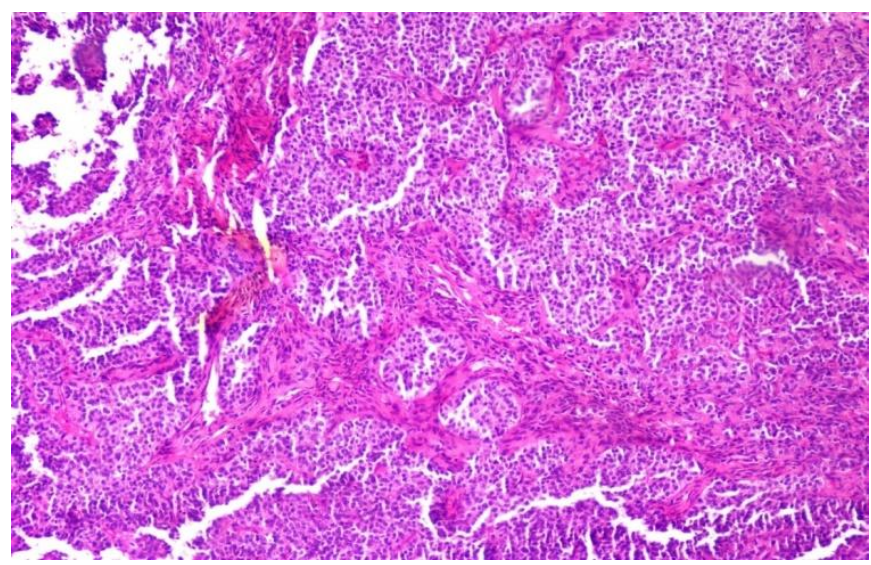

Malignant Brenner Tumor: Nests of malignant transitional cells diffusely infiltrating the fibrous stroma X 100 (H\&E) 


\section{JMSCR Vol||09||Issue||04||Page 36-46||April}

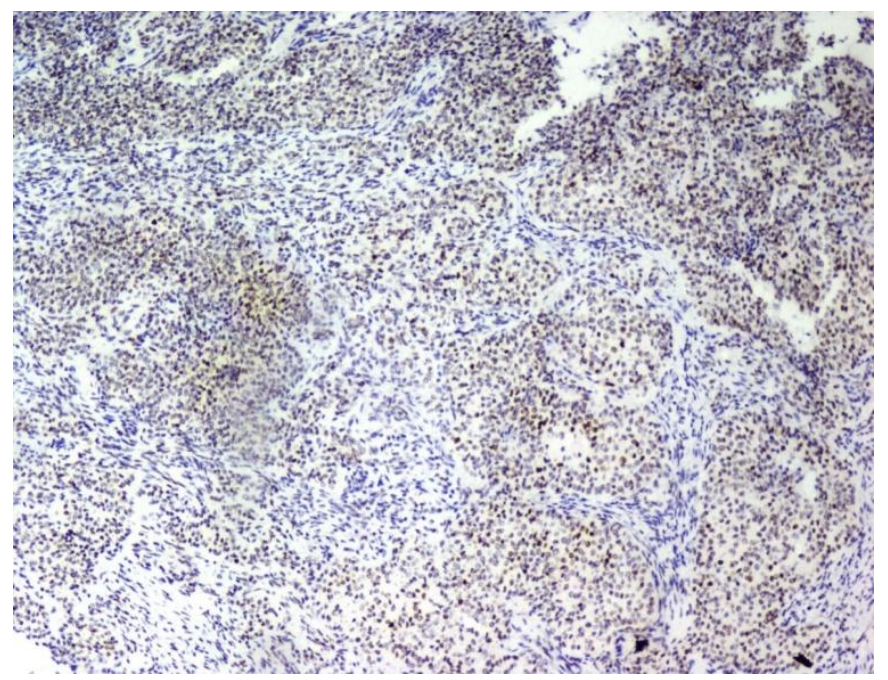

p53 Positivity In Malignant Brenner Tumor X 100

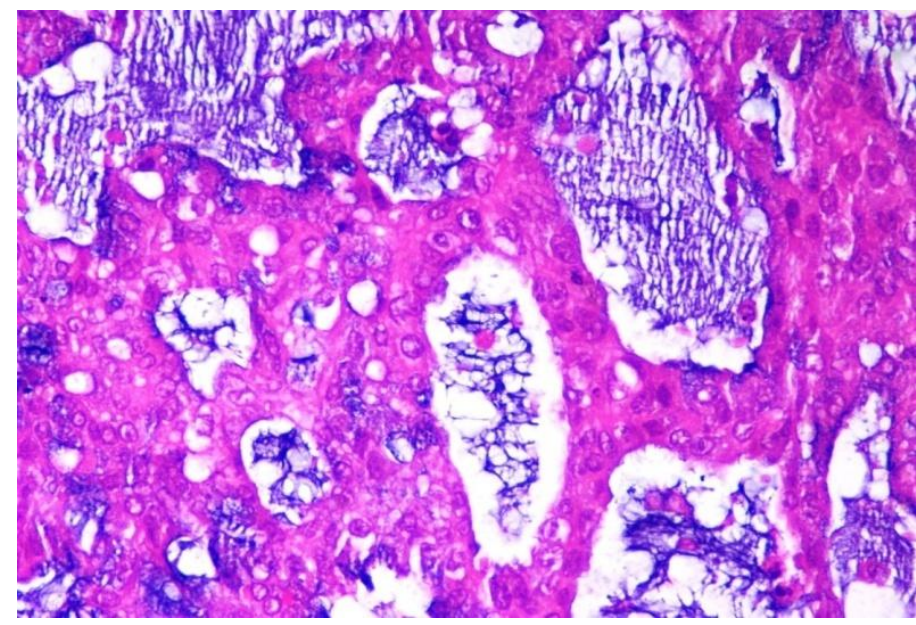

Mucinous Carcinoma: Mucin secreting malignant glands X400 (H\&E)
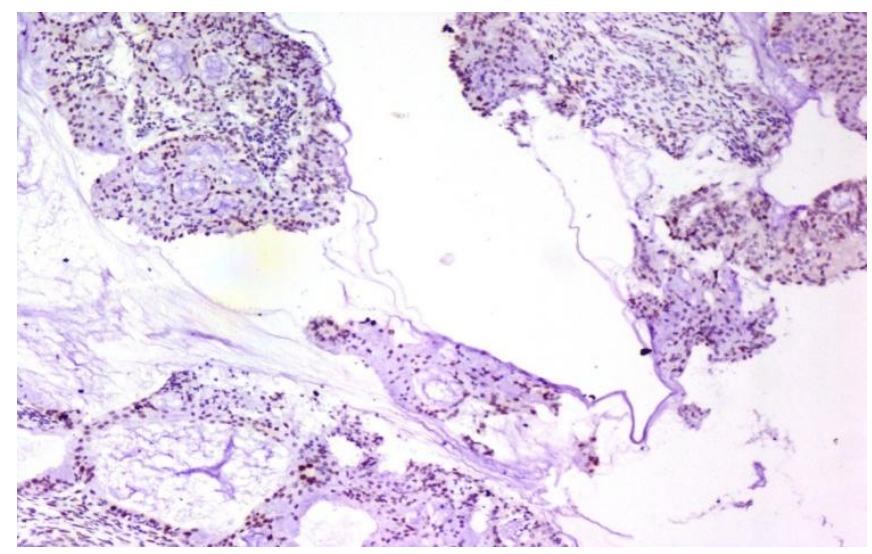

p53 Positivity in Mucinous Carcinoma (X 100)

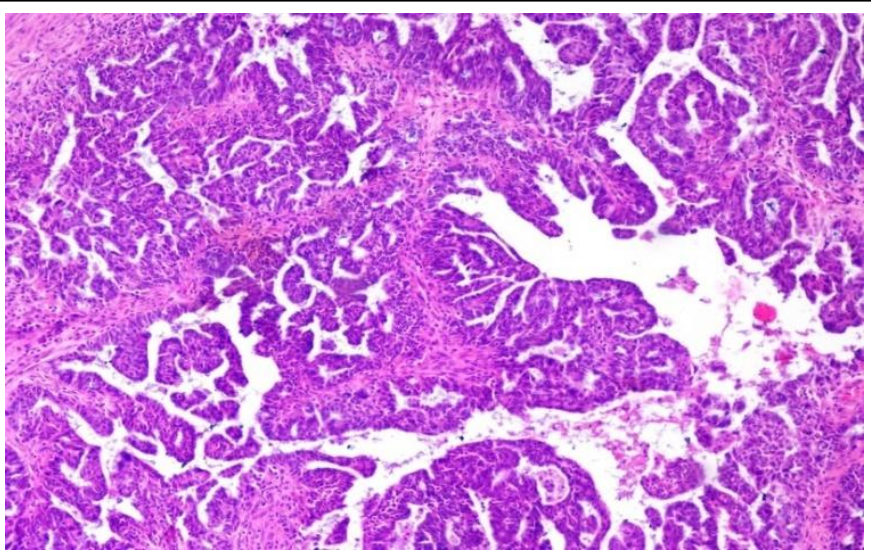

High Grade Serous Carcinoma X 100 (H\&E)

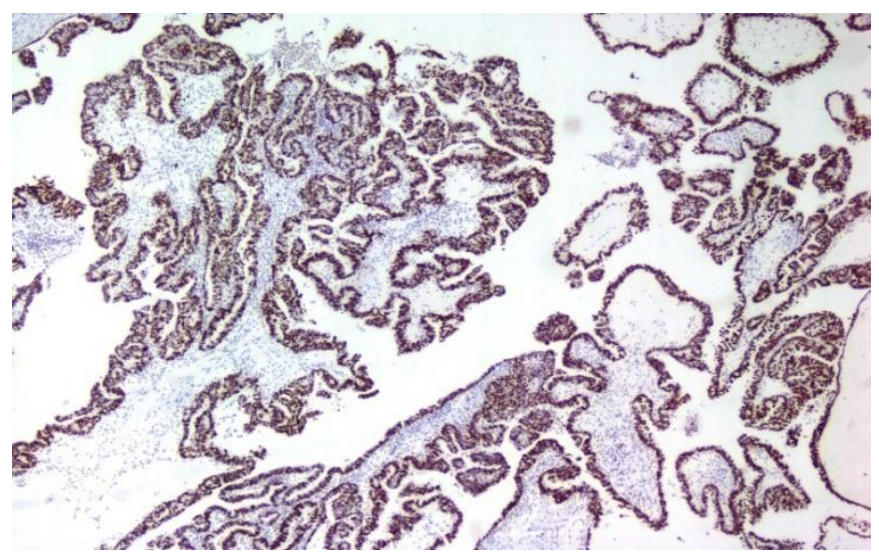

p53 Positivity In Serous Carcinoma (X 100)

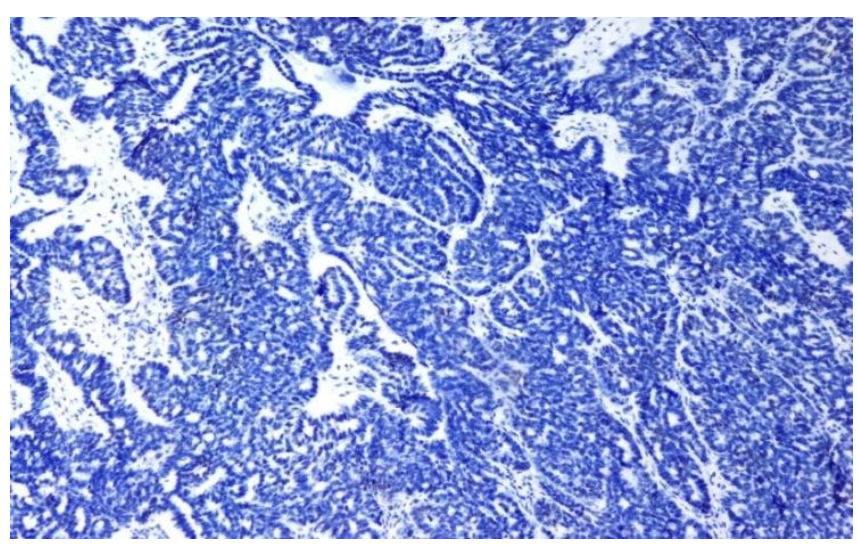

p53 Negativity In Serous Carcinoma (X 40)

\section{Discussion}

Ovarian cancer is a wide-ranging term that groups together a diverse set of neoplasms originating from the ovary, with carcinomas comprising ninety percent of ovarian cancers. Based upon morphological criteria, surface epithelial ovarian tumors (SEOTs) are classified as serous, mucinous, transitional cell, endometrioid, clear cell and mixed epithelial neoplasms. ${ }^{16}$ The etiology of SEOTs is poorly understood and 


\section{JMSCR Vol||09||Issue ||04||Page 36-46||April}

although several risk factors have been identified, their direct involvement remains largely unaddressed. Somatic and germline genetic mutations is one the important risk factor in ovarian carcinogenesis. ${ }^{17}$

p53 is the most frequently altered gene in human cancers and loss of functional p53 protein occurs in most epithelial ovarian cancers. ${ }^{18}$ Association between p53 IHC positivity and histological subtype in the literature has been controversial. Hence, there is a need to study p53 IHC in an Indian cohort considering all the technical factors that could potentially affect the staining (the antibody clone, IHC technique, interpretation of staining, etc.). ${ }^{15}$

This study evaluated the histomorphological spectrum of SEOTs and p53 expression by immunohistochemistry (IHC) in the different histological types and grades of epithelial ovarian tumors (EOT).
In the present study $44.7 \%$ of total cases were positive for p53 expression. Hamdi et al. (2012) and Tiwari et al. (2016) also found p53 expression in $43.3 \%$ and $51.2 \%$ of their total cases respectively. We found out that positive p53 expression was higher in patients with age $\geq 40$ years $(53.9 \%)$ compared to patients with age $<40$ years (25\%). Qasim et al. (2017) also noted p53 expression to be higher in patients with age $\geq 40$ years (73.2\%). However Naik et al. (2015) and Hamdi et al. (2012) found p53 positive expression to be higher in younger patients $(\mathrm{Age}=<40$ years).

We found $71.9 \%$ of malignant tumors revealed positive p53 expression followed by borderline $(50 \%)$ and benign tumors $(21.05 \%)$. Abbasi F. et al. (2016) and Qasim et al.(2017) also observed majority of malignant tumors revealing p53 expression.

Table: 1 Comparison of Results- p53 Expression and Tumor Type

\begin{tabular}{|l|c|c|c|c|c|}
\hline Authors & Year & No. of cases & Benign & Borderline & Malignant \\
\hline Naik et al. $^{\mathbf{4}}$ & 2015 & 110 & $6.1 \%$ & $75 \%$ & $75 \%$ \\
\hline Abbasi F. et al. $^{\mathbf{6}}$ & 2016 & 109 & $14.9 \%$ & $50 \%$ & $55.6 \%$ \\
\hline Qasim et al. $^{\mathbf{5}}$ & 2017 & 60 & $35 \%$ & $20 \%$ & $90 \%$ \\
\hline Chaudhary P. et al. $^{\mathbf{7}}$ & 2019 & 156 & - & $83.3 \%$ & $78.8 \%$ \\
\hline Present study & $\mathbf{2 0 2 0}$ & $\mathbf{7 6}$ & $\mathbf{2 1 . 0 5 \%}$ & $\mathbf{5 0 \%}$ & $\mathbf{7 1 . 9 \%}$ \\
\hline
\end{tabular}

Many authors suggest that the expression of p53 in benign tumors is virtually nonexistent ${ }^{4}$. However few of the studies have noted p53 positivity in benign tumors too as also reported in our study, incidence being $21.05 \%$.[Table 1]

On comparing p53 expression in different grades of malignant tumors a higher positivity was noted in low grade tumors $(100 \%)$ in the present study. This could be due to the fact that there were very less number of low grade malignant tumors reported in our study.

In the present study $45.2 \%$ serous tumors showed p53 positivity comparable to the observations by Hamdi et al. (2012). Mucinous tumors revealed positivity in less than half of the cases $(29.2 \%)$. This observation was in concordance with the results of Naik et al. (2015), Tiwari et al. (2016) and Verma R. et al. (2017).

Table: 2 Comparison of Results- p53 Expression and Histological Subtypes

\begin{tabular}{|c|c|c|c|c|c|c|}
\hline Author & $\begin{array}{c}\text { Hamdi et } \\
\text { al. }^{14}\end{array}$ & Naik et al. ${ }^{4}$ & $\begin{array}{c}\text { Tiwari et } \\
\text { al. }^{13}\end{array}$ & $\begin{array}{c}\text { Verma R. } \\
\text { et al. }\end{array}$ & $\begin{array}{c}\text { Chaudhary P. } \\
\text { et al. }^{7}\end{array}$ & $\begin{array}{c}\text { Present } \\
\text { study }\end{array}$ \\
\hline Year & 2012 & 2015 & 2016 & 2017 & 2019 & 2020 \\
\hline No. of cases & 60 & 110 & 86 & 66 & 156 & 76 \\
\hline Serous tumors & $52 \%$ & $22.4 \%$ & $80 \%$ & $27.3 \%$ & $77.8 \%$ & $45.2 \%$ \\
\hline Mucinous tumors & $62.5 \%$ & $13.3 \%$ & $43.8 \%$ & $4 \%$ & $77.8 \%$ & $29.2 \%$ \\
\hline Transitional cell tumor & $0 \%$ & $100 \%$ & $0 \%$ & $0 \%$ & Nil cases & $75 \%$ \\
\hline Endometroid tumors & $50 \%$ & $80 \%$ & $100 \%$ & $0 \%$ & $100 \%$ & $100 \%$ \\
\hline Clear cell tumors & $33.3 \%$ & $100 \%$ & $100 \%$ & $50 \%$ & Nil cases & $100 \%$ \\
\hline Seromucinous tumors & Nil cases & Nil cases & Nil cases & Nil cases & Nil cases & $0 \%$ \\
\hline
\end{tabular}


In this study seventy five percent transitional cell tumors were positive for p53 expression. As shown in the table 2 only Naik et al. in 2015 reported protein expression in all cases of transitional cell tumors. Other studies didn't find any case of transitional cell tumors.

All the cases of endometroid tumors were p53 positive as well, which is in tune with the results of Tiwari et al. (2016) and Chaudhary P. et al. (2019)

One and only case of clear cell tumor was also found to express p53 protein consistent with the results of Naik et al. (2015) and Tiwari et al. (2016). [Table 2]

In our study we found out that p53 expression was significantly higher in malignant tumors than benign tumors $(\mathbf{p}=\mathbf{0 . 0 0 0 1})$ similar to the studies carried out by of Naik et al. (2015) and Tiwari et al. (2016).p53 was expressed in higher number of low grade tumors than high grade tumors, which correlated with the study conducted by Hamdi et al. (2012).

p53 also showed higher positivity in serous, endometroid and transitional cell tumors in accordance to the studies carried out by Tiwari et al. (2016). However endometroid and transitional tumors were very less in number in our study to fairly quote the statistical significance of their p53 expression status.

\section{Conclusion}

Ovarian cancer represents the sixth most commonly diagnosed cancer among women in the world and causes more deaths per year than any other cancer of the female reproductive system. Abnormalities of p53 are the most common genetic abnormality found in malignant tumors. p53 is a nuclear protein that is involved in the control of cell cycle, apoptosis and maintenance of genomic stability. This protein can be identified by immunohistochemical technique and can be used as a surrogate marker for mutational status of p53 gene in ovarian carcinomas. ${ }^{12}$

In the present study, p53 expression was associated with older age group $(\mathbf{P}=\mathbf{0 . 0 1 8})$ and postmenopausal women $(\mathbf{P}=\mathbf{0 . 0 0 7 2})$. It was significantly higher in malignant tumors $(\mathbf{P}=\mathbf{0 . 0 0 0 1})$, serous tumors and all low grade tumors. Statistical significant correlation was not found with tumor grade $(\mathbf{P}=\mathbf{0 . 0 8 9})$ probably due to less number of cases with low grade malignancies.

To conclude, evaluation of p53 expression in surface epithelial ovarian tumors can help to better understand the biological behaviour of the tumor and formulation of new treatment modalities. Our study is limited by small sample size and follow up. Hence large sample sized studies with follow up are recommended.

\section{References}

1. T.T. Sreeja, S. Chandrasekhar, S. Lokesh Rao Magar, K. Durga, H. Sandhya Rani, S. Sandhya Anil. A Study on Expression of P53 in Surface Epithelial Ovarian Tumours. Indian Journal of Mednodent and Allied Sciences Vol. 3, No. 1, February 2015, pp- 18-25

2. RekhaVerma, Parul Gupta, NeemaTiwari, NirupmaLal, H. P. Gupta and A. N. Srivastava. Histological grade, Ca 125 levels and IHC expression of er/ pr, her2/neu, p53 and Ki 67 markers in epithelial ovarian neoplasms: a correlative study. Int. J. Adv. Res. 5(6), 235-254

3. Yi A Ren, Lisa K Mullany, Zhilin Liu, Alan J Herron, Kwong-Kwok Wong, and JoAnne S Richards. Mutant p53 promotes epithelial ovarian cancer by regulating tumor differentiation, metastasis, and responsiveness to steroid hormones. Cancer Res. 2016 Apr 15; 76(8): 2206-2218.

4. Naik PS, Deshmukh S, Khandeparkar SG, Joshi A, Babanagare S, Potdar J, et al. Epithelial ovarian tumors: Clinicopathological correlation and immunohistochemical study. J Midlife Health 2015; 6:178-83.

5. Yusra Abdulkhaliq Qasim, Shatha Zaker 
Saeed, Ilham Majeed Rashid.

Immunohistochemical Study of P53 and Ki 67 Expression in Surface Epithelial Tumor of the Ovary.Saudi J. Pathol.Microbiol.; Vol-2, Iss-3(Mar, 2017):52-59

6. Abbasi F, Esmaili A, Yekta Z, Saffarifard A. Expresion of p53 in ovaran epithelial tumours and its correlation with histopathological parameters. J Ayub Med Coll Abbottabad. 2016 Jan-Mar; 28(1):3-6.

7. Pramila Choudhary. "Role of Expression of P53 in Differentiating Benign, Borderline and Malignant Surface Epithelial Ovarian tumors." IOSR Journal of Dental and Medical Sciences (IOSRJDMS), vol. 18, no. 10, 2019, pp 37-45.

8. Dr. C. Aparna. "P53 over Expression in Ovarian Neoplasms - An Immuno Histochemical Study." IOSR Journal of Dental and Medical Sciences (IOSRJDMS) e-ISSN: 2279-0853, p-ISSN: 2279-0861.Volume 14, Issue 2 Ver. II (Feb. 2015), PP 24-27.

9. Kaur, Ashmeet \& Faujdar, Mansi \& Nakra, Sohil \& Gupta, Shubha. (2018). Histomorphological Spectrum of Bone Tumors in A Tertiary Care Hospital.Annals of Pathology and Laboratory Medicine. 5. A559-566. 10.21276/APALM.1810.

10. Swarnalatha P, Reddy SR, Chaitanya B. "Study of histomorphological spectrum of ovarian neoplasms: an institutional perspective." International Journal of Advances in Medicine | SeptemberOctober 2019, Vol 6 | Issue 5:1563-1566.

11. Phukan A, Borgogoi M, Ghosh S. "Histopathological spectrum of ovarian tumors: an institutional perspective." International Journal of Research in Medical Sciences 2018 Aug;6(8):2639-
2643

12. Dr. A.Arjunan, Dr.G.S. ThiriveniBalajji, Dr.R.Mani, Dr.Sudha.B, Dr.C.Narmadha, Dr. Vinuta Malaichamy "Expression of p53 and CD34 in Surface Epithelial Tumors of Ovary." IOSR Journal of Dental and Medical Sciences e-ISSN: 2279-0853, p-ISSN: 2279-0861.Volume 15, Issue 9 Ver. IX (September) 2016, PP 01-13

13. Tiwari Ranjan Kumar, Saha Kaushik, Mukhopadhyay Debasis, Datta Chhanda, Chatterjee Uttara, Ghosh Tarun Kumar, "Evaluation of Preoperative Serum Levels of CA 125 and Expression of p53 in Ovarian Neoplasms: A Prospective Clinicopathological Study in a Tertiary Care Hospital" The Journal of Obstetrics and Gynecology of India (March-April 2016) 66(2):107-114

14. Saleem, Shuaib \& Hamdi, Elaf. (2012). "P53 expression in ovarian tumors: (an immunohistochemical study)." Annals of the College of Medicine, Mosul. 38. 7379. 10.33899/mmed.2012.64601.

15. Amanullah NA, Poothiode U, Vilasiniamma L. "Expression of p53 in epithelial ovarian tumors." Indian J PatholMicrobiol 2020; 63:235-40.

16. Scully RE. International Histological Classification of Tumours: Histological Typing of Ovarian Tumours. 2nd edition. Heidelberg: Springer-Verlag; 1998

17. Fathalla MF. Factors in the causation and incidence of ovarian cancer.Obstetrical \& gynecological survey. 1972 Nov 1;27(11):751-68.

18. Cancer Genome Atlas Research Network. Integrated genomic analyses of ovarian carcinoma. Nature. 2011 Jun; 474 (7353): 609. 